TITLE:

\title{
A Basic Theory of Induction Heating for a Wind-Powered Thermal Energy System
}

AUTHOR(S):

Matsuo, Tetsuji; Okazaki, Toru

\section{CITATION:}

Matsuo, Tetsuji ...[et al]. A Basic Theory of Induction Heating for a Wind-Powered Thermal Energy System. IEEE Transactions on Magnetics 2017, 53(11): 8110605.

ISSUE DATE:

2017-11

URL:

http://hdl.handle.net/2433/243345

\section{RIGHT:}

(c) 2017 IEEE. Personal use of this material is permitted. Permission from IEEE must be obtained for all other uses, in any current or future media, including reprinting/republishing this material for advertising or promotional purposes, creating new collective works, for resale or redistribution to servers or lists, or reuse of any copyrighted component of this work in other works.; この論文は出版社版でありません。引用の際には出版社版をご確認ご利用ください。; This is not the published version. Please cite only the published version. 


\title{
A Basic Theory of Induction Heating for a Wind-powered Thermal Energy System
}

\author{
Tetsuji Matsuo ${ }^{1}$, Member, IEEE and Toru Okazaki ${ }^{2}$ \\ ${ }^{1}$ Graduate School of Engineering, Kyoto University, Kyoto, 615-8510, Japan \\ ${ }^{2}$ The Institute of Applied Energy, Tokyo, 105-0003, Japan
}

\begin{abstract}
A wind-powered thermal energy system can convert wind power efficiently into thermal energy to be stored for stable and low-cost electric power generation. This article derives a basic formula of induction heating for heat generation in the wind-powered thermal energy system. The dependence of heat generation on the number of magnetic poles and material parameters of the conductor is analytically derived. The maximal heat generation is given by a simple function of the conductor radius, rotational speed and applied magnetic flux density. Finite element eddy-current analysis shows that the derived analytical solution gives a reasonable estimation of heat generation.
\end{abstract}

Index Terms—Eddy current, induction heating, magnetic anisotropy, wind power

\section{INTRODUCTION}

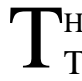
HE status of renewable energies is drastically changing. The cost of renewable energy has in some countries reduced to less than 3 cents/kWh, which is less than the cost of fossil fuel of 5 to 9 cents $/ \mathrm{kWh}$. However, renewable energies, such as wind and solar power, require power stabilization because of their intermittent nature [1], [2]. So far, this issue is solved by enhancing the power network and using backup thermal electric power plants, but this approach may not be able to be extended further because of capacity limits. Intensive research [3] on a power network showed that thermal energy storage is the most economical means of stabilizing the power network even though the efficiency of conversion from thermal energy to electric power is lower than the efficiency of conversion for batteries. An indirect thermal energy storage using an electric heater is being developed by Siemens [4]. Meanwhile, we are developing a wind-powered thermal energy system (WTES) that uses induction heating and can be realized by a light and robust rotating machine.

This paper derives a basic formula for induction heating to reveal dominant factors that determine the performance of the heat generator in the WTES.

\section{WTES}

There are two typical methods of using thermal energy storage. The first uses an electric heater. This system can be applied to any type of intermittent renewable energy output through the power network.

The second is a novel system called the WTES, which is shown in Fig. 2 [3]. Wind energy is converted to thermal energy at the top of a tower by a heat generator employing inductive heating technology. The rest of the system is the same as the concentrated solar power system. The left side of the system in Fig. 1 is operated depending on the wind condition whereas the right side is operated on demand.

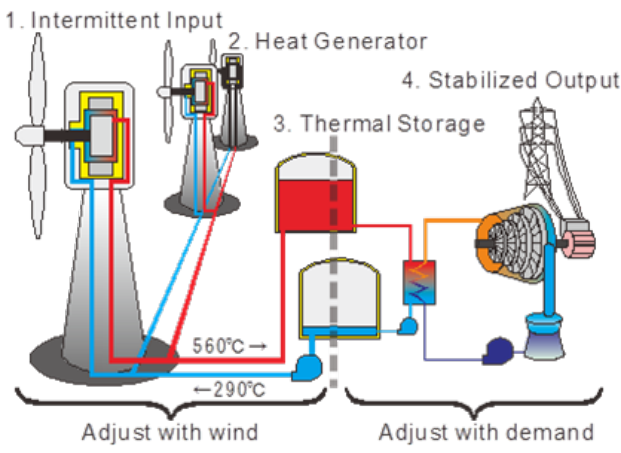

Fig. 1. Configuration of a WTES [3]

The construction cost of the wind power system is closely related to the weight of the rotating machine. A direct-drive system is superior to a geared system because of its robustness and easy maintenance. The capacity factor of the direct-drive system is much higher than that of the geared system because of the shorter downtime. However, the 2-MW direct-drive electric generator sometimes exceeds 100 tons and hence has a high total system cost owing to the construction of a strong tower. The induction heat generator employed in the WTES can be made much lighter than the electric generator because of its simplicity. The protection of permanent or electric magnets against high temperature must be also considered because part of the heat generator sometimes exceeds $600{ }^{\circ} \mathrm{C}$ [5]. From the comparison with existing similar machines such as the retarder, it is expected that the weight of inductive heat generator will be about $1 / 10$ of the direct-drive electric power 
generator while keeping the magnet temperature lower than $100{ }^{\circ} \mathrm{C}$ [3] with an air gap of several $\mathrm{mm}$. To support the estimation above and realize a light and economical heat generator, a theoretical and basic study of induction heating is required. There are a number of studies that discuss eddycurrent field and its related loss in the retarder and the rotary actuator [6]-[9]. As far as we know, however, no study gives a general formula for the heat generation to be useful in the conceptual design of WTES because the eddy-current field is geometry dependent. This study aims to provide a guideline to design the inductive heat generator from the view point of eddy-current field.
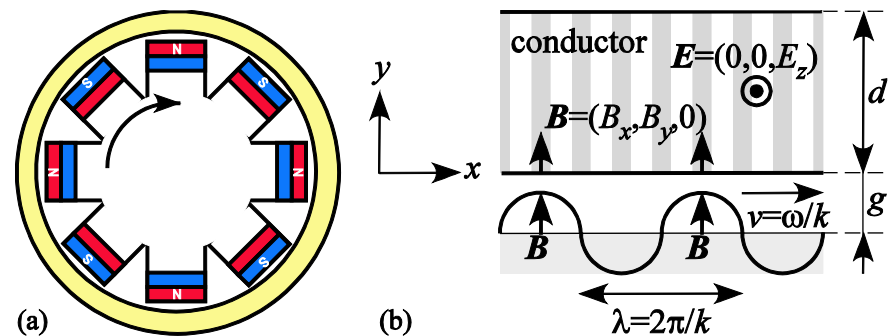

Fig. 2 Schematic of heat generation in the WTES: (a) magnetic poles and cylindrical conductor and (b) the traveling-wave approximation.

\section{BASIC THEORY OF HEAT GENERATION}

\section{A. Traveling wave approximation}

Fig. 2(a) is a schematic of an induction heating system driven by wind power, where rotating magnetic poles induce eddy current in a cylindrical conductor. For theoretical simplicity, the conductor is approximated as a plane excited by a traveling wave produced by magnet rotation as shown in Fig. 2(b). The conductor can be structured as the combination of an electric conductor and magnetic material, where the average conductivity is $\sigma$. The average magnetic property can be anisotropic with permeability $\left(\mu_{x}, \mu_{y}\right)$.

The magnetic flux density and electric field in the conductor are expressed as

$$
\begin{aligned}
& \boldsymbol{B}=\left(B_{1 x}, B_{1 y}, 0\right) \exp [\mathrm{j}(\omega t-k x)-\alpha y], \\
& \boldsymbol{E}=\left(0,0, E_{1 z}\right) \exp [\mathrm{j}(\omega t-k x)-\alpha y]
\end{aligned}
$$

where $\left(B_{1 x}, B_{1 y}\right)$ and $E_{1 z}$ are the amplitudes of the traveling wave, $k$ is the wavenumber and $\alpha$ is a complex constant. The divergence-free condition requires that

$$
B_{1 x}=-\frac{\alpha}{j k} B_{1 y} .
$$

When the number of magnet poles is $n$, the wavelength, the wavenumber and angular frequency of the traveling wave are given by $\lambda=2 \pi R /(n / 2), k=n /(2 R)$ and $\omega=n \pi f_{0}$, where $R$ is the inner radius of the cylinder and $f_{0}$ is the rotational speed.

The eddy-current field in the conductor is governed by

$$
\left(-\frac{k^{2}}{\mu_{y}}+\frac{\alpha^{2}}{\mu_{x}}\right) \boldsymbol{B}=\mathrm{j} \omega \sigma \boldsymbol{B} .
$$

From (3), $\alpha$ is given as

$$
\begin{aligned}
& \alpha^{2}=\frac{\mu_{x}}{\mu_{y}} \sqrt{k^{4}+\omega^{2} \mu_{y}^{2} \sigma^{2}} \exp (\mathrm{j} \beta), \\
& \beta=\tan ^{-1} \frac{\omega \mu_{y} \sigma}{k^{2}} .
\end{aligned}
$$

Choosing $\alpha$ having a positive real part from (4) and setting $\alpha=$ $\alpha_{R}+j \alpha_{\mathrm{I}}$, one can write as

$$
\begin{aligned}
& B_{y}=B_{1 y} \cos \left(\omega t-k x-\alpha_{\mathrm{I}} y\right) \exp \left(-\alpha_{\mathrm{R}} y\right), \\
& E_{z}=-v B_{1 y} \cos \left(\omega t-k x-\alpha_{\mathrm{I}} y\right) \exp \left(-\alpha_{\mathrm{R}} y\right)
\end{aligned}
$$

where $v=\omega / k=2 \pi R f_{0}$ is the velocity of the traveling wave. In the coordinate system moving with the traveling wave, $E_{z}$ is given as the motional electromotive force due to $-v$ and fixed $B_{y}$.

\section{B. Induction heating in a conductor}

From (5), the Joule heating is given by

$$
\sigma E_{z}^{2}=\sigma v^{2} B_{1 y}^{2} \cos ^{2}\left(\omega t-k x-\alpha_{\mathrm{I}} y\right) \exp \left(-2 \alpha_{\mathrm{R}} y\right) .
$$

Consequently, the Joule heating in the cylindrical conductor per unit width (in the $z$-direction) is

$$
W=\int_{0}^{d} \int_{0}^{2 \pi R} \sigma E_{z}^{2} \mathrm{~d} x \mathrm{~d} y=2 \pi R \frac{\sigma v^{2} B_{1 y}^{2}}{2} \frac{1-\exp \left(-2 \alpha_{\mathrm{R}} d\right)}{2 \alpha_{\mathrm{R}}}
$$

where $d$ is the thickness of the cylindrical conductor.

The skin depth of the conductor $\delta_{y}=\left(2 / \omega \mu_{y} \sigma\right)^{1 / 2}$ is much smaller than $\lambda$ practically, which means that $k^{2}<<\omega \mu_{y} \sigma$ and $\beta$ $\approx \pi / 2$. This results in

$$
\alpha_{\mathrm{R}}=\frac{|\alpha|}{\sqrt{2}}=\sqrt{\frac{\mu_{x}}{\mu_{y}}} \sqrt{\frac{\omega \mu_{y} \sigma}{2}}=\frac{1}{\delta_{x}}, \delta_{x}=\sqrt{\frac{2}{\omega \mu_{x} \sigma}} .
$$

where $\delta_{x}$ is the effective skin depth, which means that the traveling wave decays during the penetration of $\delta_{x}$.

By assuming $\delta_{x}<<d$, the Joule heating becomes

$$
W=2 \pi R \frac{\delta_{x} \sigma v^{2} B_{1 y}^{2}}{4}=R^{3} B_{1 y}^{2} \sqrt{\frac{8 \pi^{5} \sigma_{0}^{3}}{n \mu_{x}}} .
$$

The evaluation above is justified as follows. The amplitude of the motional electromotive force is $v B_{y}$, which yields Joule heat of $\sigma v^{2} B_{1 y}{ }^{2} / 2$ per unit volume. Because the skin depth for electric power is $\delta_{x} / 2$, the Joule heat in the conductor per unit length becomes $\delta_{x} \sigma v^{2} B_{1 y}{ }^{2} / 4$.

A further assumption $\delta_{x}<<\lambda$ leads to $k^{2}<<\omega \mu_{x} \sigma$, and it follows that $\left|B_{1 x}\right| \gg\left|B_{1 y}\right|$ from (2) and (8). Consequently, the approximation

$$
|\boldsymbol{B}| \approx\left|B_{1 x}\right|=\left|\frac{\alpha}{k} \| B_{1 y}\right| \approx \frac{2 R\left|B_{1 y}\right| \sqrt{\omega \mu_{x} \sigma}}{n}
$$

holds. Hence, the Joule heating in the conductor is rewritten as 


$$
W=R B^{2} \sqrt{\frac{n \pi^{3} f_{0}}{2 \sigma \mu_{x}^{3}}} .
$$

The above equation means that $\mu_{x}$ and $\sigma$ should be small because the heat is mainly generated within the effective skin depth $\delta_{x}$ given by (8). If $\mu_{x}$ and $\sigma$ are too small, however, the assumption $\delta_{x}<<\lambda$ becomes invalid.

If the magnetic poles yield higher spatial harmonic components, they can be calculated similarly to above to be superposed.

\section{Effect of the air gap}

In practice, there is an air gap between the conductor and magnetic poles, which is important for the heat protection of the magnets. The magnetic field within the air gap $(-g \leq y \leq 0$, $g$ : air gap length) is written as

$$
\boldsymbol{B}=\boldsymbol{B}_{+}+\boldsymbol{B}_{-}, \boldsymbol{B}_{ \pm}=\left(B_{ \pm x}, B_{ \pm y}, 0\right) \exp [\mathrm{j}(\omega t-k x) \pm k y]
$$

because $\sigma=0$ and hence $\alpha= \pm k$. The boundary condition at the conductor surface $(y=0)$ and the divergence-free condition $\left(B_{ \pm y}= \pm \mathrm{j} B_{ \pm x}\right)$ gives

$$
\begin{aligned}
& B_{-x}+B_{+x}=\mathrm{j}\left(B_{-y}-B_{+y}\right)=B_{1 x} / \mu_{\mathrm{r} x}, \\
& B_{-y}+B_{+y}=\left(B_{-x}-B_{+x}\right) / \mathrm{j}=B_{1 y}
\end{aligned}
$$

where $\mu_{\mathrm{rx}}=\mu_{x} / \mu_{0}$ and $\mu_{0}$ is the vacuum permeability. Let $\boldsymbol{B}$ at $y=-g$ be $\boldsymbol{B}_{\mathrm{a}}$. Using (12) and (13), $\boldsymbol{B}_{\mathrm{a}}$ is given as

$$
\left(\begin{array}{c}
B_{\mathrm{ax}} \\
B_{\mathrm{ay}}
\end{array}\right)=\left(\begin{array}{c}
B_{1 x} / \mu_{\mathrm{rx}} \\
B_{1 y}
\end{array}\right) \cosh (k g)-\mathrm{j}\left(\begin{array}{c}
-B_{1 y} \\
B_{1 x} / \mu_{\mathrm{rx}}
\end{array}\right) \sinh (k g) .
$$

The amplitude of $\boldsymbol{B}_{\mathrm{a}}$ is given by the magnetic poles. It is useful for the conceptual design of WTES to obtain a simple expression of the heat generation in terms of $\left|\boldsymbol{B}_{\mathrm{a}}\right|$. It is approximately derived by rewriting (9) using relations (2) and (14) under three conditions as follows.

(I) $k^{2}<\omega \mu_{0} \sigma / \mu_{\text {rx }}$ (or $\mu_{\mathrm{rx}} \delta_{x}<<\lambda$ ): It holds that $\left|B_{1 y}\right|<<$ $\left|B_{1 x} / \mu_{\mathrm{rx}}\right|$ and consequently $\left|\boldsymbol{B}_{\mathrm{a}}\right| \approx\left|B_{\mathrm{ax}}\right| \approx \cosh (\mathrm{kg})\left|B_{1 x}\right| / \mu_{\mathrm{rx}}$. Then, from (9) and (10), the heat generation is estimated as

$$
W=W_{1}=\cosh ^{-2}\left(\frac{n g}{2 R}\right) R B_{\mathrm{a}}^{2} \sqrt{\frac{n \pi^{3} \mu_{\mathrm{r} x} f_{0}}{2 \sigma \mu_{0}^{3}}} .
$$

The heat generation is greatly reduced when the air gap is large. Compared with (11), the dependence on $\mu_{x}$ is weak and inverted.

(II) $\omega \mu_{0} \sigma / \mu_{\mathrm{rx}}<<k^{2}<<\omega \mu_{y} \sigma$ (or $\delta_{y}<<\lambda<<\mu_{\mathrm{rx}} \delta_{x}$ ): From (9) and (14), it holds that $\left|B_{1 x}\right| / \mu_{\mathrm{rx}}<<\left|B_{1 y}\right|, \quad\left|\boldsymbol{B}_{\mathrm{a}}\right| \approx\left|B_{\text {ay }}\right| \approx$ $\cosh (\mathrm{kg})\left|B_{1 y}\right|$ and

$$
W=W_{2}=\cosh ^{-2}\left(\frac{n g}{2 R}\right) R^{3} B_{\mathrm{a}}^{2} \sqrt{\frac{8 \pi^{5} \sigma_{0}^{3}}{n \mu_{x}}} .
$$

(III) $\omega \mu_{y} \sigma<<k^{2}$ (or $\lambda<<\delta_{y}$ ): From (2) and (4), it holds that $\beta \approx 0, \alpha \approx\left(\mu_{x} / \mu_{y}\right)^{1 / 2} k$ and $\left|\boldsymbol{B}_{\mathrm{a}}\right| \approx\left|B_{1 y}\right|$. In this case, the heat generation becomes

$$
\begin{aligned}
W=W_{3} & =\cosh ^{-2}\left(\frac{n g}{2 R}\right) \frac{2 \pi \sigma R B_{1 y}^{2}}{4 k}\left(\frac{n \pi f_{0}}{k}\right)^{2} \sqrt{\frac{\mu_{y}}{\mu_{x}}} \\
& =4 \cosh ^{-2}\left(\frac{n g}{2 R}\right) \frac{\pi^{3} \sigma R^{4} f_{0}^{2} B_{\mathrm{a}}^{2}}{n} \sqrt{\frac{\mu_{y}}{\mu_{x}}}
\end{aligned}
$$

According to (16), $W$ increases with $\sigma / \mu_{x}$ as far as $\omega \mu_{0} \sigma / \mu_{\mathrm{r} x}$ $<<k^{2}$ holds, whereas (15) implies that $W$ decreases with an increase in $\sigma / \mu_{x}$ under the condition that $k^{2}<\omega \mu_{0} \sigma / \mu_{\mathrm{r} x}$. This suggests that there is an optimal value of $\sigma / \mu_{x}$ that maximizes $W$. By assuming $k^{2}<<\omega \mu_{y} \sigma$ and ignoring the phase difference between $B_{\mathrm{ax}}$ and $B_{\mathrm{ay}},\left|\boldsymbol{B}_{\mathrm{a}}\right|$ is roughly evaluated as

$$
\begin{aligned}
\left|\boldsymbol{B}_{\mathrm{a}}\right|^{2} & \approx B_{\mathrm{ax}}^{2}+B_{\mathrm{ay}}^{2} \approx \cosh ^{2}\left(\frac{n g}{2 R}\right)\left(\left|\frac{B_{1 x}}{\mu_{\mathrm{r} x}}\right|^{2}+\left|B_{1 y}\right|^{2}\right) \\
& =\cosh ^{2}\left(\frac{n g}{2 R}\right)\left(\frac{4 \pi \mu_{0} \sigma R^{2} f_{0}}{n \mu_{\mathrm{rx}}}+1\right)\left|B_{1 y}\right|^{2}
\end{aligned}
$$

Using (9) and (18), the heat generation is approximated as

$$
W=W_{4}=\frac{\cosh ^{-2}\left(\frac{n g}{2 R}\right) R^{3} B_{\mathrm{a}}^{2}}{4 \pi \mu_{0} R^{2} f_{0} \sqrt{\frac{\sigma}{\mu_{\mathrm{rx}}}}+n \sqrt{\frac{\mu_{\mathrm{rx}}}{\sigma}}} \sqrt{\frac{8 n \pi^{5} f_{0}^{3}}{\mu_{0}}}
$$

which is a combined expression for conditions (I) and (II) because it is approximated by (15) or (16) when $k^{2}<<$ $\omega \mu_{0} \sigma / \mu_{\mathrm{rx}}$ or $\omega \mu_{0} \sigma / \mu_{\mathrm{rx}}<<k^{2}$, respectively. The heat generation (19) has the maximal value

$$
W_{\max }=\cosh ^{-2}\left(\frac{n g}{2 R}\right) \frac{\pi^{2} R^{2} f_{0} B_{\mathrm{a}}^{2}}{\sqrt{2} \mu_{0}}
$$

when

$$
\frac{\mu_{\mathrm{r} x}}{\sigma}=\frac{4 \pi \mu_{0} R^{2} f_{0}}{n} .
$$

Equation (21) means that $k^{2}=\omega \mu_{0} \sigma / \mu_{\mathrm{rx}}$ and consequently that $\left|B_{\mathrm{ax}}\right|=\left|B_{\mathrm{ay}}\right|$ at the conductor surface under the assumption of $k^{2}$ $<\omega \mu_{y} \sigma$. Even though $B_{x}$ does not induce the motional electromotive force, it cannot be small to obtain the maximal heat generation. This is due to the energy flow from the magnetic poles to the conductor, which is evaluated by the $y$ component of Pointing vector in the moving coordinate system: $S_{y}=E_{z} H_{x}=-v B_{y} B_{x} / \mu_{0}$.

If the air gap is sufficiently small, $W_{\max }$ becomes

$$
W_{\max }=\frac{\pi^{2} R^{2} f_{0} B_{\mathrm{a}}^{2}}{\sqrt{2} \mu_{0}} .
$$

When $f_{0}=0.25 \mathrm{~Hz}, R=2.5 \mathrm{~m}$ and $B_{\mathrm{a}}=1 \mathrm{~T}, W_{\max }$ becomes 8.8 MW. Equation (22) (or (20)) is useful to estimate the generator size to yield a required heat generation. The optimal conductor material can be chosen from the condition (21) depending on the number of poles. Equation (20) clearly shows the dominant factors to determine the heat generation. The evaluation (22) does not contain the material parameters and the number of poles because it is roughly due to $S_{y}=$ 
$-v B_{y} B_{x} / \mu_{0}$ with $v=2 \pi f_{0} R$.

\section{Finite ELEMENT EDDY-CURRENT ANALYSIS}

This section performs a numerical simulation of the eddycurrent field. The equation for the magnetic potential $A$ :

$$
\frac{\partial}{\partial x}\left(\frac{1}{\mu_{y}} \frac{\partial A}{\partial x}\right)+\frac{\partial}{\partial y}\left(\frac{1}{\mu_{x}} \frac{\partial A}{\partial y}\right)=\sigma \frac{\partial A}{\partial t}
$$

is solved by the finite element method (FEM) using linear triangular elements. The half spatial period including one pole is divided into 20 segments along the $x$-direction whereas the air-gap and the conductor are divided into 10 and 40 segments, respectively, along the $y$-direction; $R$ and $f_{0}$ are set at $2.5 \mathrm{~m}$ and $0.25 \mathrm{~Hz}$, respectively. A sinusoidal traveling wave of $B_{\text {ay }}$ is applied such that the maximal $\left|\boldsymbol{B}_{\mathrm{a}}\right|$ is $1 \mathrm{~T}$.

The steady-state filed given by the FEM exactly agree with the analytical field solution, e.g. (14), derived in the preceding section. Fig. 3 depicts the flux lines yielded by the finite element method where $n=8, \sigma=1 \times 10^{6} \mathrm{~S} / \mathrm{m}$ and $\left(\mu_{\mathrm{rx}}, \mu_{\mathrm{ry}}\right)=$ $(2,1000)$; the air gap is located at $-0.1 \leq y \leq 0 \mathrm{~m}$. The flux lines slant considerably in the conductor $(0 \leq y)$, which means that $B_{y}$ is less than $B_{x}$ that does not contribute to induction heating directly. This may restrict the Joule heating because high $B_{x}$ prevents $B_{y}$ from being high within the limitation of $\left|\boldsymbol{B}_{\mathrm{a}}\right|$.

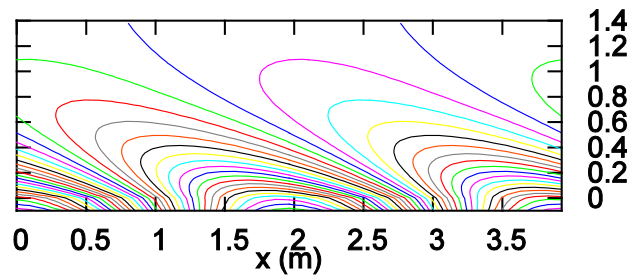

$y(m)$

Fig. 3 Flux lines: $n=8$ and $R=2.5 \mathrm{~m}$.

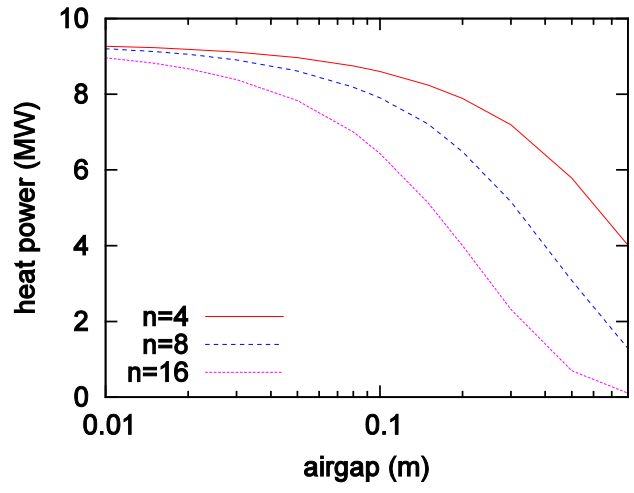

Fig. 4 Effect of the air gap with $n=4,8,16$.

Fig. 4 shows the dependence of heat generation on the air gap when $\left(\mu_{\mathrm{rx}}, \mu_{\mathrm{ry}}\right)=(2,1000)$ and $(n, \sigma)=\left(4,5 \times 10^{5} \mathrm{~S} / \mathrm{m}\right),(8$, $\left.1 \times 10^{6} \mathrm{~S} / \mathrm{m}\right)$ and $\left(16,2 \times 10^{6} \mathrm{~S} / \mathrm{m}\right)$. Several $\mathrm{cm}$ of air gap does not decrease the heat generation significantly. This is helpful for the heat protection of magnet. The effect of the air gap becomes large when $n$ is large because of the effect of $\cosh ^{-2}(n g / 2 R)$ appearing in (15)-(17) and (19). However, it should be taken into account that the decrease in $n$ increases the skin depth $\delta_{x}$ requiring large conductor thickness $d$.

Fig. 5 compares the computed heat generation with the analytical solutions $W_{1}, W_{2}, W_{3}$ and $W_{4}$, where $n=8, g=0.01$ $\mathrm{m}, \sigma=1 \times 10^{6} \mathrm{~S} / \mathrm{m}$ and $\left(\mu_{\mathrm{r} x}, \mu_{\mathrm{ry}}\right)=(2,1000),(1,1)$. Estimation $W_{1}$ is valid when $\sigma$ is large where $\delta_{x}$ is small. When $\sigma$ is small, estimation $W_{2}$ or $W_{3}$ is valid under the condition (II) that $\delta_{y}<<$ $\lambda$ [Fig. 5(a)] or condition (III) that $\lambda \ll \delta_{y}$ [Fig. 5(b)], respectively. Approximation $W_{4}$ gives a reasonable estimation when $\delta_{y}<<\lambda$ [Fig. 5(a)]. Condition (III) is not recommended because a value of $\sigma$ that is too small reduces $W$ and increases the skin depth, thus requiring large conductor thickness $d$.

Fig. 6 shows the heat generation property with the air gap $g$ $=0.1 \mathrm{~m}$ depending on (a) $(n, \sigma)$ and (b) $\left(\mu_{x}, \mu_{y}\right)$. Fig. 6(a) shows that the heat generation decreases with an increase in $n$ because of the effect of the term $\cosh ^{-2}(n g / 2 R)$. The optimal conductivity proportionally increases with $n$ according to (21). Fig. 6(b) shows that $\mu_{y}$ has only a small effect on heat generation. This is predicted by (19), which does not include $\mu_{y}$. The optimal $\mu_{x}$ is small so as not to reduce the effective skin depth $\delta_{x}$.

When $\boldsymbol{B}$ is produced by the exciting current, $\mu_{y}$ should be large so as to reduce the exciting current by decreasing the magnetic resistance of the conductor, even though $\mu_{y}$ does not affect $W_{4}$. In this case, the magnetic anisotropy of $\mu_{x}<<\mu_{y}$ is recommended, and it can be realized by a stripe structure perpendicular to the $x$-direction where nonmagnetic conductors are sandwiched by magnetic materials, for example. The stripe structure may increase the heat generation by concentrating the Joule heat in the conductor, which will be addressed in future work.

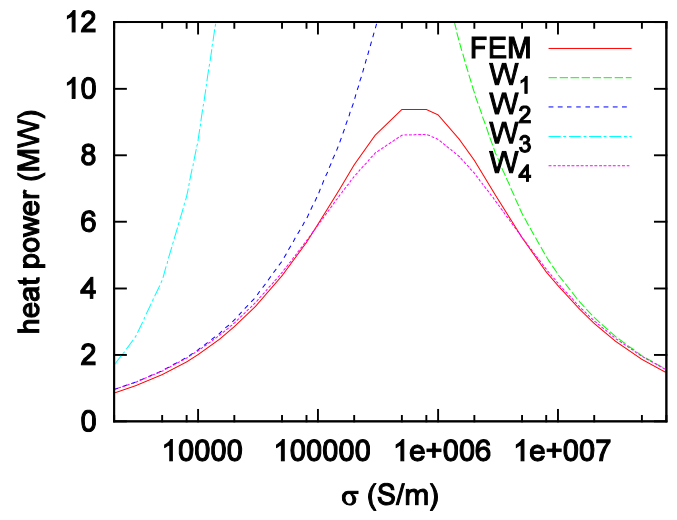

(a)

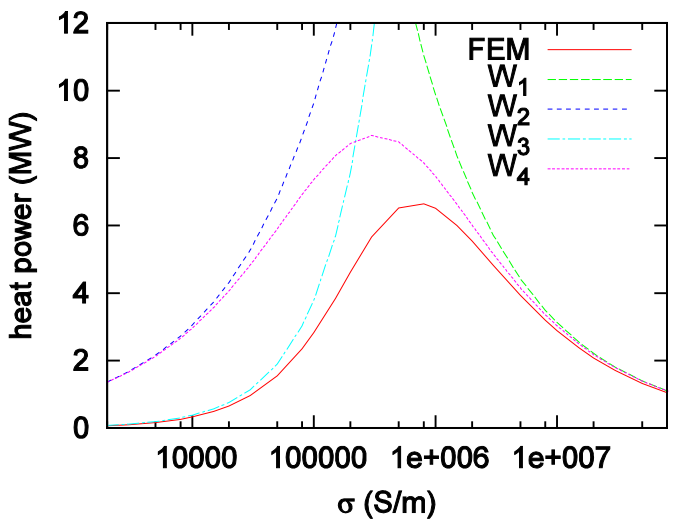

Fig. 5 Effect of conductivity with $n=8$ and $g=0.01 \mathrm{~m}$ : (a) $\left(\mu_{\mathrm{rx}}, \mu_{\mathrm{ry}}\right)=(2$, $1000)$ and $(\mathrm{b})\left(\mu_{\mathrm{rx}}, \mu_{\mathrm{ry}}\right)=(1,1)$. 
(a)

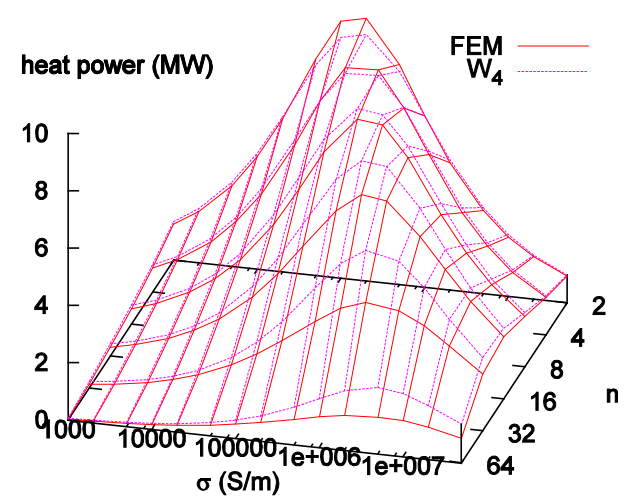

(b)

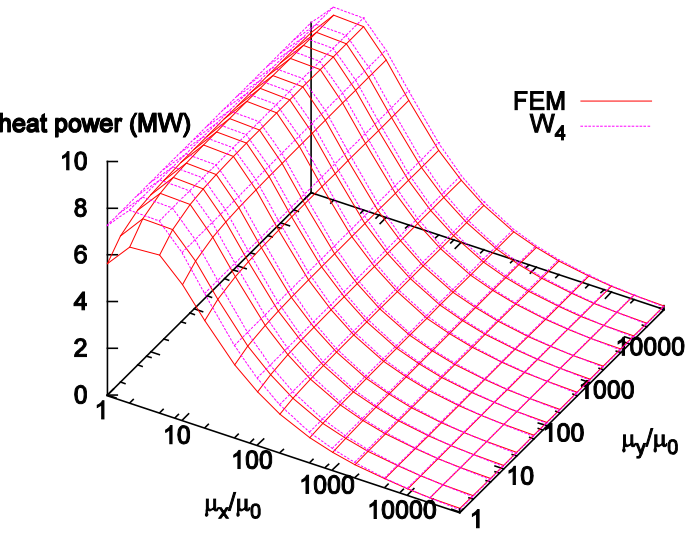

Fig. 6 Heat generation property: (a) dependence on $(n, \sigma)$ when $\left(\mu_{\mathrm{rx}}, \mu_{\mathrm{ry}}\right)=(2$, $1000)$ and (b) dependence on $\left(\mu_{\mathrm{rx}}, \mu_{\mathrm{ry}}\right)$ when $(n, \sigma)=\left(8,1 \times 10^{6} \mathrm{~S} / \mathrm{m}\right)$.

\section{CONCLUSION}

A basic formula of induction heating was analytically derived to reveal the dependence of heat generation on the number of magnetic poles and the conductivity and permeability of the conductor. The maximal heat generation is given by a simple function of the conductor radius, rotational speed, applied magnetic flux density, number of poles and air gap length, revealing the dominant factors of induction heat generation in the WTES. The derived condition to maximize the heat generation can be used to choose optimal conductor material. Using the heat generation estimation, a thermal analysis will be performed in future.

\section{ACKNOWLEDGMENT}

The author T.M. would like to express his gratitude to Mr. J. Izawa and Mr. S. Omura, B.S. students at Kyoto University, Japan, for their assistance in finite element analysis.

\section{REFERENCES}

[1] M. Arita, A. Yokoyama, Y. Tada, "Evaluation of battery system for frequency control in interconnected power system with a large penetration of wind power generation,” Int. Conf. on Power System Tech., Chong qing, Chi., Oct. 2006.

[2] International Energy Agency, "Medium-term renewable energy market report 2016,” ISBN PRINT 978-92-64-26496-0.

[3] T. Okazaki, Y. Shirai, T. Nakamura, "Concept study of wind power utilizing direct thermal energy conversion and thermal energy storage," Renewable Energy, vol. 83, pp. 332-338, Nov. 2015.

[4] Siemens. (Sept. 2016). Press Release: Siemens presents thermal storage solution for wind energy. [online]. available: https://www.siemens.com/press/pool/de/pressemitteilungen/2016/windp ower-renewables/PR2016090419WPEN.pdf.

[5] Y. Noguchi, M. Miyahara, K. Imanishi, A. Saitoh, N. Kishine, "Creepfatigue life prediction for permanent magnet type eddy current retarder," Proc. 8th Intl. Fatigue Congress, vol. 5, pp. 3101-3018, Jun. 2002.

[6] K. Muramatsu, N. Takahashi, T. Hashio, C Yamada, M. Ogawa, S. Kobayashi, T. Kuwahara, "3-D eddy current analysis in moving conductor of permanent magnet type retarders using moving coordinate system,” IEEE Trans. Energy Convers., vol. 14, pp. 1312-1317, Dec. 1999.

[7] M. Markovic and Y. Perriard, "An analytical determination of eddy current losses in a configuration with a rotating permanent magnet," IEEE Trans. Magn., vol. 43, pp. 3380-3386, Aug. 2007.

[8] J.J.H. Paulides, K.J. Meessen, E.A. Lomonova, "Eddy-current losses in laminated and solid steel stator back iron in a small rotary brushless permanent-magnet actuator,” IEEE Trans. Magn., vol. 44, pp. 43734376, Nov. 2008.

[9] C.Y. Liu, K.J. Jiang, Y. Zhang, "Design and use of an eddy-current retarder in an automobile,” Int. J. Automot. Tech., vol. 12, pp. 611-616, 2011. 